\title{
La rebelión de Huánuco, 1812: rumores, fiestas y espacio cotidiano
}

\section{The Huánuco rebellion of 1812: rumors, parties and daily space}

\author{
Marissa Bazán Díaz \\ Universidad de Lima
}

\section{RESUMEN}

El presente artículo estudia la rebelión de Huánuco de 1812 , considerando que los sectores populares tuvieron la capacidad de utilizar una cultura política propia. Para lograrlo, se prestó atención al papel que desempeñaron los rumores sobre la situación que atravesaba el virreinato por la ausencia del rey Fernando VII, las fiestas como el carnaval y el espacio público donde se difundió un discurso oculto de crítica política. De esta manera, se reflexionará sobre las aspiraciones que llevaron a levantarse a los rebeldes contra las autoridades españolas, más allá de solo los dirigentes criollos, logrando rescatar parte de las expectativas políticas de la población del común.

\section{Palabras clave:}

Proceso de Independencia; rebelión de Huánuco, cultura política indígena; rumores y fiestas

\section{ABSTRACT}

This article studies the Huánuco rebellion of 1812, considering that popular sectors were able to use a political culture of their own. To achieve this, attention was paid to the role played by rumors about the situation that the viceroyalty was going through because of the absence of King Ferdinand VII, parties such as the carnival, and the public space where a "hidden discourse" of political criticism was spread. In this way, this research will reflect on the aspirations that led the rebels to rise against the Spanish authorities, beyond just the creole leaders, managing to rescue part of the political expectations of the common population.

Keywords:

Independence process, Huánuco rebellion, indigenous political culture, rumors, parties 
L a rebelión de Huánuco de 1812 estalló durante la fiesta del carnaval, treinta años después del levantamiento de Túpac Amaru II, apareciendo previo a su inicio una gran cantidad de rumores mediante los cuales se convocó a la población (Peralta, 2012, p. 321; Varallanos, 1959, pp. 474-475). Aunque el resultado fue el fracaso de la causa rebelde, tras apenas un mes de su inicio, esta insurrección mostró parte de las expectativas políticas propias de dichos pueblos. La principal causa del levantamiento fueron las disposiciones procedentes de Lima, de índole económica, las cuales prohibieron la venta de tabaco y de productos agrícolas, lo que afectó a criollos e indios respectivamente (Dunbar Temple, 1971, p. XXXVIII; Glave, 2008, p. 392). Sin embargo, estas medidas no resultaron contrarias a los intereses de los europeos, por ejemplo, la familia Llanos —emparentada con el subdelegado de Huánuco, Diego García- mantuvo sus privilegios.

De esta manera, miles de indios marcharon, tomaron el puente de Huayaupampa e iniciaron varios saqueos en Huánuco, Ambo, Llata, entre otros pueblos, con la finalidad de presionar para que se produjera el cese de dichas medidas fiscales y denunciar los abusos de las autoridades. Si bien esta situación económica y política fue la causa principal del alzamiento, para el presente trabajo - gracias a la perspectiva de la cultura política-, se decidió considerar los rumores y las fiestas como elementos claves para enriquecer la comprensión de lo ocurrido en Huánuco como parte de las luchas regionales del proceso de independencia, tan necesario de conocer camino hacia la conmemoración de su bicentenario oficial (Aljovín y Jacobsen, 2007, pp. 13-83).

\section{El papel de los rumores en la rebelión}

La rebelión de Huánuco comenzó el 22 de febrero de 1812, pero previamente circularon rumores de su próximo estallido, tal como lo comunicó el intendente Gonzales Prada en un oficio dirigido al virrey, el 5 de marzo de ese año, en el cual señaló que doce días antes de la invasión a esta ciudad, en Lima ya se conocía dicha intención. Por ello, el cura Jado denunció la incapacidad del subdelegado Diego García, ya que no tomó las medidas necesarias pese a saber sobre dicha noticia, y a esto, se sumó su huida a Cerro de Pasco, abandonando Huánuco (Eguiguren, 1938, p. 11). Pero, ¿un rumor puede ser tomado cómo una información cierta? Si la respuesta es positiva, la responsabilidad del ataque — que incluso terminó en saqueos, muertes y actos como el entierro de una mujer viva, la danza sobre hombres degollados y la antropofagia-, pudo haberse prevenido.

En primer lugar, para reconocer cómo los rumores se convierten en importantes e influyentes es considerar que estos contienen una información vinculada a sucesos cotidianos o a alguna inquietud puntual que debe ser de interés público. Además, suelen aparecer frente a la falta de noticias oficiales, bajo la presión de la ansiedad y del miedo que las personas puedan tener de cara a los hechos, por lo que su exactitud o falsedad no se pueden verificar inmediatamente. Entonces, para que el rumor tenga impacto social, debe poseer por lo menos dos condiciones: importancia y ambigüedad, existiendo la posibilidad de ser la versión extraoficial de los hechos (Allport y Postman, 1967, pp. 15 y 25). Por ello, contienen parte de la verdad, siendo "una suerte de noticia improvisada con el consenso colectivo" (Fernández, 2012, pp. 197-200)' . Justamente, estos elementos dieron pie a la circulación de rumores en Huánuco, los que adquirieron mucha importancia, porque creemos que alentaron a los rebeldes a decidirse por el camino del alzamiento con la finalidad de acabar con la situación injusta e incomprensible por la que estaban atravesando.

Las medidas fiscales afectaron a toda la población huanuqueña, excepto a los europeos, siendo este un tema relevante. $Y$ los anuncios extraoficiales no se dejaron esperar en medio de un tiempo confuso — debido a la crisis de la monarquía, la formación de las Juntas de Gobierno y la ausencia de un medio informativo oficial-, que conllevó varias interpretaciones en el virreinato peruano, lo cual resultó en rumores convincentes para muchos de los futuros alzados y en una manera de acceder a la información de los hechos. De esta forma, si bien las disposiciones comerciales y los abusos de las autoridades fueron importantes para tomar posición, los rumores complementaron dicha decisión siendo utilizados por todos los niveles sociales como una estrategia o instrumento del poder, al punto que en aquella época no solo los incógnitos, sino el

1 Cuando existen situaciones críticas, como guerras o rebeliones, la ambigüedad se apodera de la situación con fuerza (Fernández, 2012, pp. 199-200; Scott, 2004, pp. 176, 178 y 179). El libro de Lefebvre demuestra esos deseos y miedos como factores en "la Grande Peur". 
propio Estado, los esgrimía para lograr controlar a sus oponentes (Scott, 2004, p. 175) ${ }^{2}$. En el caso del subdelegado García, se comprende que les haya dado poca importancia, ya que se trataba de una información extraoficial que rechazaba las medidas que él más bien defendía, difundida por los sectores populares.

En segundo lugar, los rumores son básicamente orales, y la oralidad es el canal de difusión más utilizado por los sectores populares de mayoría iletrada y carentes de voz oficial. Esos rumores, al transmitirse de boca en boca, tergiversaban los hechos, acrecentaban el miedo y agravaban la visión de los acontecimientos (Luna, 2011, p. 138). Debido a ello, a esta parte de la población, los rumores le sirvieron como un medio para desestabilizar aquello que consideraron inadecuado de los gobiernos, sobre todo en épocas de guerra, siendo una manera de cuestionar el orden y la organización establecida (Almeida, pp. 3-12). Incluso para el gobierno y la élite, esta forma de transmisión se catalogó como más peligrosa que la libertad de prensa, ya que favorecía a la multiplicación de noticias falsas repercutiendo de manera relevante entre aquellos que los asumían como verdades (Lefebvre, 1986, p. 103; Scott, 2004, pp. 192-193; Almeida y Ojeda, p. 2). De allí que existía la preocupación del Estado por controlarlos, y desde el siglo xVIII, tenemos que la Inquisición fue clave en este cometido, aunque no logró evitar la difusión de las nuevas ideas que llegaron por contrabando. En el caso de los espacios públicos, se los vigiló mediante una especie de policía secreta, buscando impedir el llamado a la sedición. Ciertamente, es más fácil prohibir un periódico oficial que detener cartas o pasquines ocultos y evocaciones orales, a partir de las cuales las palabras circulan y se construyen interpretaciones sin el control del poder de turno (Chassin, 2013, p. 408; Rosas, 2006, p. 79).

Justamente, Huánuco en su intento de frenar la situación de abusos, utilizó la difusión de boca en boca de la información, en la que se denunciaban estos hechos y las posibles soluciones, lo que resultó clave para el alzamiento. Uno de sus rumores principales tuvo conexión con la Junta de Buenos Aires, la cual, consciente de la importancia de la difusión de noticias para reclutar rebeldes, encargó a Castelli que enviara emisarios a las provincias del Perú como Cusco, Puno, Arequipa, Tacna, bajo la lógica de que "hay que siempre tener a la indiada de su lado"; y mensajeros conocidos como "forasteros" o "seductores", los que posibilitaron difundir el rumor de que pronto iba a llegar a Huánuco "el Rey Castel, el Rey Inca", posiblemente como una alternativa al vacío de poder (Chassin, 2013, pp. 408-411; Peralta, 2012, p. 323) ${ }^{3}$. Un testigo de la época, Manuel Carrillo, vecino de Huánuco, comerciante español, de 30 años, indicó:

que solo dos veces ha conversado sobre Casteli, primero ahora cinco meses poco mas o menos con don Narciso Bracamonte, por haverle contado este, que el Señor Goyeneche lo había derrotado a dicho Casteli, embiado las banderas a Lima, y celebrado Misa en gracias, a que le contexto con alegría que estaba bueno, cuya conversación se ofreció en casa del finado Don Agustín Pérez. Segunda con José Beraun a quien le dixo, que havia oído decir que venía Casteli por Mainas, expresándole que era un disparate semejante noticia, lo que adquirio de oídas entre Indios, y responde. (CDIP, tomo III, Vol. 2, p. 152)

La llegada de Castelli como inca generó varias expectativas que se propagaron de boca en boca y en pasquines (la forma escrita de colocar los rumores). Pero no fue lo único que se difundió: otra información extraoficial anunció que a la casa del subdelegado don Diego García habría llegado un supuesto capellán, perteneciente a un navío inglés, con varios cuadernos, y que este capellán había señalado a la población "que ya venía toda la nación Bretánica a sogusgar a Huánuco y su territorio" (CDIP, Tomo III, Vol. 1, p. 258). Los rumores fueron difundidos en forma de versos, con los cuales se anunció la muerte de Fernando VII, la negativa de apoyar la soberanía de la Junta de Regencia, el buscar derrotar a los chapetones, la idea de contar con una América separada del dominio español, entre otros. Frente a esto, resultó favorable exaltar los hechos revolucionarios de Buenos Aires, Tucumán, Chile y el Alto Perú; informar que Santa Fe y Caracas se habían sublevado; y que algo similar ocurriría en el virreinato peruano, sobre todo, por contar con el respaldo de Río de la Plata. Mediante la difusión de esta información, se provocaron — con sus verdades incompletas - expectativas de un posible triunfo o, por lo menos, de una pronta mejora de su situación (Dunbar Temple, 1971, pp. LIV-LV) ${ }^{4}$.

2 Godenzzi analizó los pasquines de la rebelión de Tupac Amaru II desde el punto de vista semántico, destacando el estudio de "tiranía" (Rosas, 2006, pp. 180-196; 2008, pp. 156-162).

3 Lo peculiar es que en el momento de la rebelión de Huánuco Castelli ya había sido derrotado.

4 Para profundizar lo que estaba ocurriendo en esta época en el Alto Perú, consultar el trabajo de Glave (2008, pp. 380-387). 
De esta manera, los rumores fueron tomados probablemente como creíbles, por un gran número de rebeldes que buscaron comprender lo que estaba aconteciendo, encontrando información de su interés, posibles salidas a su situación injusta y explicaciones a sus confusiones debido a la carencia de noticias oficiales (Guerin y Miyazaki, 2003). Los rumores al difundirse en las conversaciones cotidianas cobraron una función que permitió el conocimiento de los hechos a los sectores populares, aunque principalmente conspirativos, por lo que su difusión se realizó a escondidas. Su objetivo fue incitar a la insurgencia para destruir a los tiranos chapetones, tal como denunciaba fray Ignacio Villavisencio. También, permitieron convencer a varios pobladores para que se unieran voluntariamente bajo la expectativa de mejoras; por ello, estos discursos fueron un instrumento político importante para que se produjera la rebelión de Huánuco ${ }^{5}$.

\section{Los rumores y las fiestas}

Los rumores actuaron de manera relevante al complementar la decisión de apelar a la rebelión, pues posibiltaron la captación de una mayor cantidad de adeptos, antes y durante el alzamiento. Uno de los espacios cotidianos por donde se los difundió fueron las fiestas. Bajo esta situación, tenemos la declaración de don Mariano Cárdenas, natural de la ciudad de Cuenca, ubicado en el reino de Santa Fe — al cual se le dio la fama de alzado en el pueblo-, quien el día 26 de febrero de 1811, fecha en la que se celebra el Corpus, narró lo siguiente:

Contestó que nada había sobre lo que le hacía cargo, y que si allí se habían partido alguna palabras habría sido efecto dela mucha vevida... por haver cantado unas Desimas de la Concordia en el espresado día de Corpus se le notó revoltoso y alsado... no es posible que por cantar unos versos patrióticos se le acuse de poco consequente a la obediencia debida a él Rey á la Patria... lo que deve expresar es que las décimas que allí se cantaron en el citado día, no fueron patrióticas, y si contrarias; pues según se acuerda de los quatro primeros versos, dicen así: El Chapetón y el criollo, se unieron en amistad, con la misma intimidad, que un Gavilán con un Pollo; y responde. (CDIP, Tomo III, Vol. 3, p. 196)

Con motivo de la fiesta y un año antes de que se desatara la rebelión, el declarante nos revela, en primer lugar, que la idea de recurrir a las acciones rebeldes para cambiar el escenario de abusos estaba preparándose con bastante tiempo; en segundo lugar, estos rumores hicieron uso de figuras como los "gavilanes", para denunciar a los chapetones que cometían injusticias fingiendo una falsa amistad con "los pollos", los cuales representaron a los criollos. Durante el virreinato, el calificar a las personas utilizando animales, resultó en varios casos una forma de violencia cotidiana, por lo que probablemente se esgrimió así este discurso para simbolizar la extensión de los acontecimientos que se buscaron denunciar (Geertz, 2003, p. 358; Torres, 2016, p. 207). Además, a esta reunión asistieron varios vecinos, como don Agustín Sambrano y don Domingo Moreno con sus esposas y don José Tello y don Manuel Peña (CDIP, Tomo III, Vol. 3, p. 198), quienes estaban celebrando el Corpus, una fiesta religiosa: esto permitió que los rumores y el discurso conspirador pudieran difundirse de manera pública, a diferencia de la privacidad de otros espacios como la casa o algún lugar clandestino.

Otra declaración fue la del teniente de milicias don Antonio de Zavala, quien dijo que estando como "Alcalde en la Montaña me hicieron denuncia estos de que Antonio Espinosa el Limeño havia bosiferado públicamente en el asiento de Mesapata un día de fiesta, estando congregados a oir Misa, expresiones de insurreccion" (CDIP, Tomo III, Vol. 1, p. 382); y la de don Juan Garcia, quien señaló "Que hallándome en la Montaña de Chinchau, subcedió que Antonio Espinoza Vociferó públicamente un día de fiesta contra los Chapetones, diciendo barios improperios contra ellos y amenazando que en breve vendría Casteli a acabar con todos ellos" (CDIP, Tomo III, Vol. 1, p. 383). Ambos, muestran cómo las

5 "El rumor se diferencia del chisme en tanto cruza o atraviesa las barreras de los grupos sociales y no solo versa sobre asuntos de terceras personas. Existen rumores de mayor y de menor extensión, cuya circulación se limita a un contexto cultural y rumores que atraviesan distintas culturas a veces hasta continentes" (Zires, s. f., p. 175). “El rumor es un pariente cercano del chisme y de la agresión mágica. Aunque no tenga necesariamente como objeto a una persona en particular, se trata de una poderosa forma de comunicación anónima que puede servir a intereses muy específicos [...] la mejor situación para que prosperen los rumores es cuando ocurren acontecimientos de vital importancia para los intereses populares y sólo se tiene acceso a información ambigua o definitivamente dudosa [...] Los acontecimientos en que hay peligro de perder la vida, como las guerras, las epidemias, las hambrunas y los motines, son en general los marcos sociales más fértiles para la producción de rumores [...] La autonomía y la volatilidad del rumor político pueden fácilmente provocar actos violentos [y es muy rápido en su propagación, por ello la necesidad de los poderosos de reprimirlo, como señala Ranajit Guha]" (Scott, pp. 168-175; Fernández, 2012, p. 201). 
fiestas y las misas de celebración fueron usadas como espacios para incitar al levantamiento, al aglutinar a varias personas y posibilitar así la captación de una mayor cantidad de receptores, a los cuales se buscó animar a alzarse mediante la difusión de estos rumores. Además, desde el 20 de enero de 1812, un mes antes de la rebelión, en la fiesta religiosa de San Sebastián, circularon rumores en los pasquines - dentro y fuera de Huánuco-, contra los vecinos europeos. El ayudante mayor de las milicias de infantería de la ciudad de Huánuco, don Francisco Ingunsa, declaró:

Que desde el dia veinte de Enero en que se celebra la fiesta de San Sebastían Patrón de la ciudad de Huánuco, empezaron a ponerse Pasquines contra los vecinos Europeos, diciendo estas palabras, Quatro Provincias tenemos: estaos a la primera vez para acabar con ellos, esto es con los Europeos, y otros en lengua india con multiplicación de ellos... Que ulteriormente, en principios del presente mes se retiraron repetidos mas Pasquines referentes a que daban tres oras de termino a los Europeos para que saliesen de dicha ciudad... diciéndole que la gente estaba próxima pronta... que también supo por oídas que un hombre Indio, ya venerable, cuyo nombre se ha ignorado, anduvo por el Pueblo de Santa Maria del Valle, Pillao, Acomayo y otros inmediatos a la jurisdicción de Huánuco y Panataguas, repartiendo papeles, reducidos a convocar a los Pueblos, a que se reuniesen en Huánuco a las ocho de la mañana del dia veintitrés. (CDIP, Tomo III, Vol. 1, pp. 84-85)

La fiesta de San Sebastián también fue utilizada para hacer la difusión de los rumores e incitar al alzamiento y no solo conmemorar hechos sagrados; sin embargo, estas amenazas se hicieron más intensas en la siguiente festividad: los carnavales. Eulalio Paredes, cuyo oficio era el de sombrerero, residente de Huánuco, en su declaración señaló:

Que este inició por Pasquines puestos contra los Europeos: que aseguran se abibó el calor contra estos porque un Don N. Quintana el martes del carnaval maltrató gravemente á un moso criollo de Huánuco a merito de no haver querido darle un palo, que llevaba estando en una cachua ... Que asi duraron estos rumores hasta el savado veinte, $y$ uno del ultimo febrero, en que apareció un Alcalde de Yndios de esos pueblos inmediatos, mostrando al subdelegado una orden, que suponía haver mandado citar a esos dichos pueblos Comarcanos por un incognito... que ha oydo posteriormente, que de esos trese pueblos reveados se han retirado muchos Yndios apesarados de sus hechos, y otros se mantie/nen hasta ver las resultas, creyendo que ya $\mathrm{ni}$ manda el Señor Virrey de Lima, sino Casteli... (CDIP, Tomo III, Vol. 1, pp. 118-120)

Cabe mencionar que el carnaval, otra de las fiestas traídas por los europeos, se desarrollaba durante los tres días previos a la Cuaresma, por lo que era una especie de despedida de los placeres terrenales, y mientras duraba, eran permitidas actividades mundanas como "conductas irreverentes, bailes sensuales, palabras soeces, disfraces grotescos, el consumo excesivo de vino y comida, alusiones al sexo y representaciones que ridiculizaban a las autoridades" (Rojas, 2005, p. 31). Por tanto, en esta celebración se podía ridiculizar a las autoridades, y fue precisamente durante esta que en Huánuco se inició el estallido de la rebelión, encontrando cierto grado de permisividad para probablemente denunciar los abusos de los poderosos, porque a los participantes se les permitía imaginar la inexistencia de las jerarquías. Contrario a esto, las fiestas religiosas oficiales buscaron, más bien, recordar las diferencias, por lo que se cree que el inicio de la rebelión durante su festejo no fue una simple coincidencia.

Además, dicha lógica, no fue completamente ajena al imaginario del mundo andino. No se debe olvidar que se creía en la existencia del pachacuti, el cual, si bien no era igual a la propuesta carnavalesca, proponía coincidentemente la posibilidad de crear un mundo al revés ${ }^{6}$. De esta manera, probablemente la dinámica de esta última fiesta, brindó el ambiente propicio que incentivó el inicio de la rebelión con el objetivo de volver realidad las aspiraciones de cambio de los rebeldes. La difusión de los rumores que se llevó a cabo desde un año antes, lo cual nos muestra que no se trató de un alzamiento improvisado, fue bastante trascendental por su poder de convocatoria, y contribuyó, junto con la fiesta, a la organización del levantamiento, a partir de la convocatoria pública a quienes aspiraban a destruir a los chapetones abusivos.

6 "A diferencia de la fiesta oficial, el carnaval era el triunfo de una especie de liberación transitoria, la abolición provisional de las relaciones jerárquicas, privilegios, reglas y tabúes Se oponía a toda perpetuación, a todo perfeccionamiento y reglamentación, apuntaba a un provenir aún incompleto. La abolición de las relaciones jerárquicas poseía una significación muy especial. En las fiestas oficiales, las distinciones jerárquicas se destacaban a propósito, cada personaje se presentaba con las insignias de sus títulos, grados y funciones, y ocupaba el lugar reservado a su rango. Esta fiesta tenía por finalidad la consagración de la desigualdad, a diferencia del carnaval en el que todos eran iguales y donde reinaba un contacto libre y familiar entre individuos normalmente separados en la vida cotidiana por las barreras infranqueables de su condición, su fortuna, su empleo, su edad y su situación familiar" (Bajtín, 1994, pp. 15-16). Sobre el pachacuti puede consultar a Flores, 1988, pp. $42-61$. 


\section{Referencias}

\section{Fuentes primarias}

La Revolución de Huánuco, Panataguas y Huamalíes. Colección Documental de la Independencia del Perú 1, 2, 3, 4, 5 (III) Lima: Comisión Nacional del Sesquicentenario de la Independencia del Perú.

\section{Fuentes secundarias}

ALJovín, C. y JACOBSEN, N. (2007). En pocas y en muchas palabras: una perspectiva prágmatica de las culturas políticas, en especial para la historia moderna de los Andes. En C. Aljovín y N. Jacobsen (Eds.), Cultura política en los Andes (1750-1950). Lima: IFEA/Universidad nacional Mayor de San Marcos-Fondo Editorial.

Allport, W. y Postman, L. (1967). Psicología del rumor. Buenos Aires: Editorial Psique.

AlmeidA, M. y OJeDA, C. (s. f.). Análisis del discurso del rumor como escenario en el contexto conformador de la opinión pública de la sociedad quiteña. Caso: la asamblea constituyente del año 2007 (tesis de licenciatura, Universidad Politénica Salesiana, Quito, Ecuador). Recuperado de http://dspace.ups.edu.ec/ bitstream/123456789/2428/1/ANALISIS\%20DEL\%20DISCURSO\%20DEL\%20RUMOR\%20COMO\%20 ESCENARIO\%20EN\%20EL\%20CONTEXT.pdfhttp://dspace.ups.edu.ec/bitstream/123456789/2428/1/ ANALISIS\%20DEL\%20DISCURSO\%20DEL\%20RUMOR\%20COMO\%20ESCENARIO\%20EN\%20EL\%20 CONTEXT.pdf

BAJTín, M. (1994). La cultura popular en la Edad Media y el Renacimiento. El contexto de François Rabelais. Madrid: Alianza.

ChASSIN, J. (2008). El rol de los alcaldes de indios en las insurrecciones andinas (Perú a inicios del siglo xIx. Bulletin de I'Institut Francais d'Études Andines 1(37).

ChASSIN, J. (2013). Guerra de información y guerra de propaganda entre Lima y Buenos Aires (1808-1812). En S. O'Phelan Godoy \& G. Lomné (Eds.), Abascal y la contraindependencia de América del Sur. Lima IFEA/ Pontificia Universidad Católica del Perú-Fondo Editorial.

Dunbar Temple, E. (1971). Conspiraciones y rebeliones en el siglo xix. La Revolución de Huánuco, Panataguas y Huamalíes. Colección Documental de la Independencia del Perú 1(III). Lima: Comisión Nacional del Sesquicentenario de la Independencia del Perú. I-XCVII.

Eguiguren, L. (1938). Segunda tentativa de rebelión de Huánuco (1813). Revista Universitaria II, Año VIII.

FernÁNDEZ, A. (mayo-agosto del 2012) Psicología de masas, identidad social, epidemias y rumores: la influenza en México. Sociológica, año 27, (76), 189-230. Recuperado de http://www.scielo.org.mx/pdf/soc/v27n76/ v27n76a6.pdf

GeERTZ, C. (2003). La interpretación de las culturas. Barcelona: Gedisa Editorial.

GLAVE, L. (julio-setiembre del 2008). Cultura política, participación indígena y redes de comunicación en la crisis colonial. El Virreinato Peruano, 1809-1814. Historia mexicana LVIII(1). El Colegio de México, 369-426.

GueRIN, B. y MiYAZAKI, O. (2003). Rumores, chisme y leyendas urbanas: una teoría de contingencia social. Revista Latinoamericana de Psicología, 35(3), 257-272. Recuperado de http://www.redalyc.org/ pdf/805/80535302.pdf

LefeBvRE, G. (1986). El gran pánico de 1789. La Revolución francesa y los campesinos. Buenos Aires: Editorial Paidós.

LUNA, C. (julio-diciembre del 2011). La política desde los circuitos de comunicación en la Provincia de Cartagena, 1830-1839. Historelo. Revista de Historia Regional y Local, 3(6), 127-152. Medellín: Universidad Nacional de Colombia. Recuperado de http://www.redalyc.org/articulo.oa?id=345832079006 
Peralta, V. (2012). La participación en las juntas de gobierno peruanas de Huánuco (1812) y Cuzco (1814). En P. Cagiao Vila, P. y J. Portillo Valdés (Eds.), Entre imperio y naciones. Iberoamérica y el Caribe en torno a 1810. España: Universidad de Compostela.

Rojas, R. (2005). El ascenso de lo popular a la cultura nacional (Lima, 1822-1922). Lima: Instituto de Estudios Peruanos. Recuperado de http://books.openedition.org/ifea/5089?lang=es

RosAs, C. (2006). Del trono a la guillotina. El impacto de la Revolución Francesa en el Perú (1789-1808). Lima: Instituto Francés de Estudios Andinos/Pontificia Universidad Católica del Perú-Fondo Editorial.

Rosas, C. (2008). El miedo a la revolución. Rumores y temores desatados por la Revolución Francesa en el Perú, 1790-1800. En C. Rosas (Ed.), El miedo en el Perú. Siglo xvı al xx. Lima: Pontificia Universidad Católica del Perú-Fondo Editorial.

ScotT, C. J. (2004). Los dominados y el arte de la resistencia. Discursos ocultos. México: Cultura Libre.

Torres, E. (2016). La violencia en los Andes: historia de un concepto, siglos XVI-XVII. Lima: Pontificia Universidad Católica del Perú/Instituto Riva-Agüero. Recuperado de //repositorio.pucp.edu.pe/index/handle/ 23456789/54125

VARAllanos, J. (1959). Historia de Huánuco. Introducción para el estudio de la vida social de una región del Perú. Desde la época prehistórica hasta nuestros días. Buenos Aires: Imprenta López.

ZiRES, M. (s. f.). Las dimensiones del rumor: oral, colectiva y anónima. Recuperado de http://148.206.107.15/ biblioteca_digital/capitulos/29-920kwu.pdf P. 175.

ZIRES, M. (1998). El rumor y los poderes locales. Voces y Cultura. Revista de Comunicación. Barcelona. 